\title{
A Randomized Clinical Trial of Synbiotics in Irritable Bowel Syndrome: Dose- Dependent Effects on Gastrointestinal Symptoms and Fatigue
}

Sang-Hoon Lee', Doo-Yeoun Cho², Seok-Hoon Lee', Kyung-Sun Han', Sung-Won Yang', Jin-Ho Kim', Su-Hyun Lee', Soo-Min Kim ${ }^{1}$, Kyu-Nam Kim ${ }^{1, *}$

'Department of Family Practice and Community Health, Ajou University School of Medicine, Suwon, Korea

${ }^{2}$ Department of Clinical Pharmacology and Therapeutics, CHA Bundang Medical Center, CHA University School of Medicine, Seongnam, Korea

\section{See editorial commentary page on 1}

Background: This double-blind, randomized controlled design study aimed to assess the dose-dependent effects of synbiotics on gastrointestinal symptoms of and fatigue in irritable bowel syndrome (IBS).

Methods: Thirty subjects with IBS were randomly assigned into the following three groups and received 2 capsules a day for 8 weeks: (1) high-dose ( 2 capsules of synbiotics); (2) low-dose (1 capsule of synbiotics and 1 capsule of placebo); and (3) placebo (2 capsules of placebo). At baseline and 8 weeks, they completed the study questionnaires.

Results: Two subjects in the high-dose group were lost to follow-up, leaving a total of 28 patients for the analysis. After 8 weeks, abdominal discomfort, abdominal bloating, frequency of formed stool, fatigue Visual Analog Scale (VAS), and Multidimensional Fatigue Inventory were significantly different among the groups $(\mathrm{P}=0.002,0.006$, $0.007,0.028$, and 0.041 , respectively, by Kruskal-Wallis test). However, only abdominal discomfort, abdominal bloating, frequency of formed stool, and fatigue VAS were significantly improved in the high-dose group compared with those in the placebo group ( $\mathrm{P}=0.002,0.003,0.002$, and 0.013 , respectively) by Mann-Whitney test with Bonferroni correction. No adverse drug reactions were reported.

Conclusion: High-dose synbiotics were superior to placebo in improving bowel symptoms and fatigue of IBS patients, suggesting that synbiotic dosage plays an important role in the treatment of IBS.

Keywords: Irritable Bowel Syndrome; Synbiotics; Fatigue; Dose-Response Relationship; Probiotics

Received: May 16, 2017, Revised: June 15, 2017, Accepted: June 16, 2017

${ }^{*}$ Corresponding Author: Kyu-Nam Kim https://orcid.org/0000-0002-1213-5004

Tel: +82-31-219-5309, Fax: +82-31-219-5218, E-mail: ktwonm@hanmail.net 


\section{INTRODUCTION}

Irritable bowel syndrome (IBS) is a functional gastrointestinal (GI) disorder characterized by abdominal pain, abdominal discomfort, and altered bowel habits. ${ }^{1)}$ The prevalence of IBS is reported as approximately $5 \%-10 \%$ of the general population with a female predominance in South Korea. ${ }^{2)}$ It has a negative impact on health-related quality of life and induces high health costs in Asian populations as well as Western countries. ${ }^{3}$

Although the precise etiology of IBS is still unknown, emerging evidence suggests that gut flora play a pathological role. ${ }^{4,5)}$ The corollary to this theory is the possible benefit of probiotics for IBS symptoms, and several meta-analyses have concluded that probiotics containing specific strains do improve the global symptoms of IBS and abdominal pain. ${ }^{6,7)}$ Therefore, recent interest has focused on agents such as proand prebiotics whose actions are confined to the lumen, with a perceived low likelihood of unwanted systemic effects in IBS patients. In theory, certain pro- and prebiotics, at a correct dose with the appropriate formulation, can help restore the balance of the intestinal microbiota, leading to better digestive and intestinal function and possibly improved GI symptoms. A mixture of probiotics and prebiotics, namely synbiotics or symbiotics, should exert a synergistic benefit by enhancing the probiotic organisms with a selective and co-administered prebiotic substrate. Increased consumption of synbiotic supplements may also have synergistic or potentiating effect in terms of health benefits. However, data regarding the dose-response relationship between synbiotics and IBS symptoms are scarce. In addition, because disruption of gut microbial balance may aggravate quality of life in IBS, supplementation with synbiotics may help resolve fatigue symptoms in
IBS patients.

Thus, we investigated the dose-response effects of synbiotic supplementation on bowel symptoms and fatigue in IBS patients.

\section{METHODS}

\section{Study Design and Subjects}

This study was a single-center, randomized, double-blind, and placebo-controlled clinical trial. Among the subjects who visited the health promotion center of Ajou University Hospital from June 2015 to October 2015, those who met the Rome III criteria for IBS were enrolled in this study (Figure 1). IBS patients aged $\geq 19$ years were recruited, and initial screening included medical history taking, physical examination, and laboratory testing. We excluded subjects who met one of the following conditions: subjects with organic GI disease such as inflammatory bowel disease and a history of gluten intolerance (celiac disease); subjects taking antibiotics, synbiotics (including probiotics only), or any other drug that could influence bowel function within 1 month of consumption; the subjects with previous abdominal surgery except for appendectomy; pregnant or breastfeeding females; subjects with evidence of other organic, metabolic, or psychiatric diseases that could impact compliance as well as those with poor medication compliance.

Of the 38 subjects who underwent the screening process, eight applicants were excluded according to the inclusion/exclusion criteria. Therefore, 30 subjects were initially enrolled in the study; of these, 28 subjects completed this study, as two were withdrawn for unknown reasons.

All the subjects completed an informed consent form before partici-

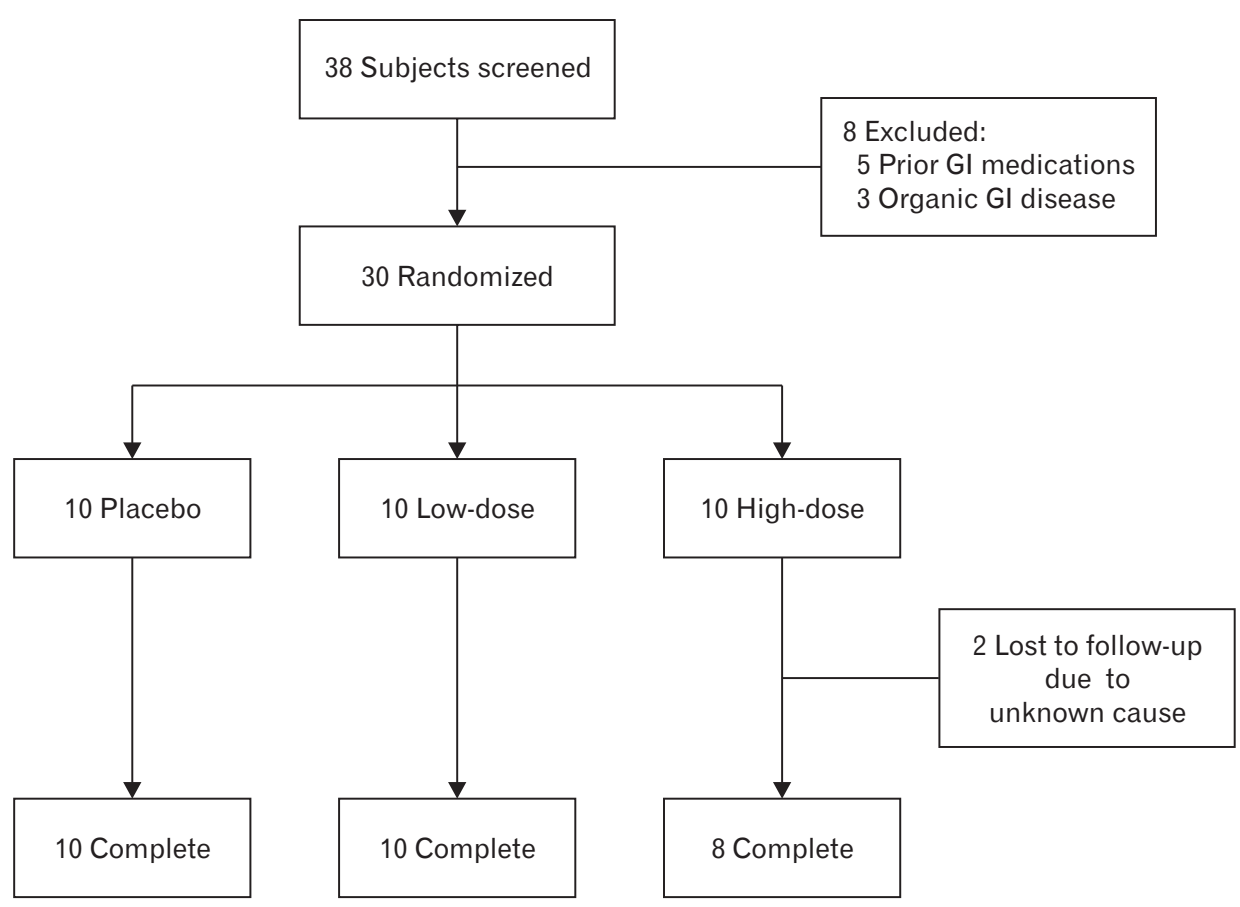

Figure 1. Study flow diagram. Gl, gastrointestinal. 
pating in the study, and the study was conducted after obtaining approval from the institutional review board of Ajou University Hospital (AJIRB-MED-DRU-14-317). This study was conducted in compliance with the standards of the Declaration of Helsinki and current ethical guidelines.

\section{Study Protocol \& Randomization}

The subjects were randomly assigned at a 1:1:1 ratio to the high-dose, low-dose, or placebo groups according to block randomization. We did not control any variables during randomization. The patients, investigators, doctors, and study nurses were blinded using randomization codes, which were kept confidential until the end of data analysis. The placebo group took 2 capsules of placebo, the low-dose group received 1 capsule of placebo and 1 capsule of synbiotics, and the highdose group received 2 capsules of synbiotics. The study drugs were administered once a day for 8 weeks. All patients filled in fatigue and bowel symptom questionnaires at the first and last visit. After 4 weeks of the study, all subjects were revisited and their physical condition, vital signs, study compliance, and adverse events were evaluated. Their laboratory test results, study compliance, and adverse events were then re-checked after the completion of 8 weeks of the study. During the entire study period, all patients were prohibited from consuming yogurt or taking pre- or probiotic pills or other GI drugs.

\section{Sample Size Estimation}

The size of the study sample was not based on a power calculation owing to the absence of reliable calculation data about synbiotics in IBS. Instead, it was considered adequate to characterize a potential effect with sufficient accuracy based on previous experience gained through similar studies. ${ }^{8-10)}$ In other words, the previous two studies were performed with 25 subjects and 24 subjects with a double-blind randomized design, respectively. ${ }^{8,10)}$ In another single-blind randomized controlled trial (RCT) of 30 patients that investigated the effects of probiotics on intestinal permeability in IBS, ${ }^{9}$ the authors estimated that the sample size of 30 patients had a power of $80 \%$ to detect a difference of $30 \%$ in the proportion of increased small bowel permeability between the placebo and the probiotics group after treatment at the significance level of $5 \%$ with a low dropout rate $(<5 \%)$.

\section{Synbiotics \& Placebo Preparation}

The investigational drug was Ultra-Probiotics-500, supplied by B\&A Health Products Inc., Los Angeles, CA, USA. Each capsule contains 10 billion colony-forming units of probiotic bacteria comprising six strains of Lactobacillus (rhamnosus, acidophilus, casei, bulgaricus, plantarum, and salivarius) and two strains of Bifidobacterium (bifidum and longum). Each capsule also contains $175 \mathrm{mg}$ of fructooligosaccharides, $150 \mathrm{mg}$ of Ulmus davidiana (Slippery elm bark powder), $10 \mathrm{mg}$ of Geum urbanum (herb bennet) powder, and $100 \mathrm{mg}$ of inulin powder as prebiotics. The placebo was encapsulated in the same material as the synbiotic capsule, and the content of the capsule was manufactured such that it could not be distinguished by taste or color.

\section{Data Collection}

\section{1) Blood sampling}

Blood samples were drawn after midnight fasting. We checked the complete blood cell count and tested liver function by examining the levels of aspartate aminotransferase (AST), alanine aminotransferase (ALT), and gamma-glutamyltranspeptidase (GGT) and renal function by assessing the levels of blood urea nitrogen (BUN) and creatinine.

\section{2) IBS Symptom Questionnaire}

The primary endpoints included severity of the Visual Analog Scale (VAS) scores for IBS individual symptoms such as abdominal discomfort, abdominal bloating, frequency of formed stool, epigastric soreness, and nausea. The VAS symptom scores consisted of a 10-point scale, with 0 indicating no symptoms and 10 indicating very severe or very frequent symptoms. The adjectival scales for stool consistency (Bristol Stool Form Scale) were recorded as numerical values. ${ }^{11)}$ The data on the VAS scores of individual symptoms were summarized as the mean and standard deviation in each treatment group.

\section{3) Fatigue Questionnaire}

To assess the degree of fatigue symptoms in IBS patients, we used the Fatigue Severity Scale (FSS) ${ }^{12)}$ The FSS contains nine items, each of which is ranked for degree of severity using a scale of 1 (none) to 7 (very severe); the score is the average of the nine items. We also used the fatigue VAS to assess the degree of global fatigue, using a scale of 0 (no fatigue) to 10 (very severe fatigue). Additionally, we used the 20item Multidimensional Fatigue Inventory (MFI) as a self-report instrument for measuring degree of fatigue severity. ${ }^{13)}$ It addresses the following dimensions: general fatigue, physical fatigue, mental fatigue, reduced motivation, and reduced activity. All the items were scored on a 5-point Likert scale ranging from 1 (yes, that is true) to 5 (no, that is not true). Subscale scores (range, 4-20) were calculated as the sum of item ratings, and the total fatigue scores (range, 20-100) were calculated as the sum of subscale scores, with higher scores indicating a higher level of fatigue.

\section{Statistical Analysis}

The data were examined using intention-to-treat (ITT) and per-protocol (PP) analyses. For the ITT analysis, all data from the study subjects were included. For missing or incomplete data, the most recently available data were used for a last-observation-carried-forward (LOCF) analysis. For the PP analysis, only the data obtained from the subjects who completed the study on schedule were included, and the analysis was performed only when the data were available at weeks 0 and 8 . Of the 30 subjects who enrolled in the study, two subjects were withdrawn (from the high-dose group) and none were omitted due to noncompliance. All side effects were reported and noted in detail, regardless of their relevance to the study drug.

Descriptive statistics and a frequency analysis were performed to describe the clinical characteristics of the study subjects. We used the 
Wilcoxon signed rank test to calculate the difference in clinical variable scores between the baseline and after the study within the groups. Then, we used the Kruskal-Wallis test for the comparison of mean clinical characteristics scores between the three groups at baseline and the end of the study (placebo, low-dose, and high-dose groups). We also performed the Kruskal-Wallis test to compare the differences in variables from baseline at 8 weeks among the groups. A P-value $<0.05$ was considered to indicate statistical significance. For variables with a significant difference between the three groups in the Kruskal-Wallis test at the end of study, we performed the Mann-Whitney test with Bonferroni correction. According to Bonferroni correction of the three groups, P-values $<0.017$ were considered to indicate statistical significance. Subsequently, we used the Spearman rank correlation test to establish the dose-response trends. All analyses were carried out using IBM SPSS ver. 19.0 (IBM Corp., Armonk, NY, USA).

\section{RESULTS}

\section{Baseline Characteristics}

Table 1 shows the baseline clinical characteristics of the study subjects in the 3 groups by PP analysis ( $n=28$ ). Overall, the mean age of the study population was $50 \pm 8.6$ years, the median age was 50.5 years, and $53.6 \%$ of the study population was female. The average duration of IBS was $11 \pm 9.5$ years, and the main subtype was the diarrheal subtype at
$71.4 \%$ (constipation subtype was $21.4 \%$, mixed subtype was $7.1 \%$ ). The average abdominal discomfort score was $5.4 \pm 1.7$, abdominal bloating score was $5.6 \pm 2.2$, fatigue VAS was $4.5 \pm 1.9$, and MFI was $89.3 \pm 13.1$.

There were no significant differences between the three groups with respect to gender, abdominal discomfort, abdominal bloating, frequency of formed stool, epigastric soreness, and nausea; only the age of the subjects showed differences $(\mathrm{P}=0.086,0.286,0.255,0.384,0.506$, 0.399 , and 0.020 , respectively). The differences in age (and gender proportion, even it was not significant) at baseline were considered a random error due to the small numbers of subjects in each study group. In addition, there were no significant differences between the groups in terms of baseline biochemistry characteristics, including white blood cell count, hemoglobin, platelet count, AST, ALT, GGT, BUN, and creatinine $(\mathrm{P}=0.231,0.452,0.612,0.893,0.115,0.569,0.492$, and 0.500 , respectively). All these values were within the normal range. The mean FSS, VAS, and MFI scores were not significantly different between the groups at baseline ( $\mathrm{P}=0.609,0.256$, and 0.438 , respectively).

\section{Changes in Variables after Intervention}

Abdominal discomfort, abdominal bloating, fatigue VAS, and MFI in the low-dose and high-dose groups were significantly improved after intervention ( $\mathrm{P}=0.005,0.007,0.008$, and 0.008 in the low-dose group; $\mathrm{P}=0.010,0.011,0.012$, and 0.011 in the high-dose group, respectively), but not in the placebo group ( $\mathrm{P}=0.271,0.180,0.196$, and 0.681 ) by $\mathrm{PP}$

Table 1. Baseline characteristics of the study subjects $(n=28)$

\begin{tabular}{|c|c|c|c|c|}
\hline Characteristic & Placebo $(n=10)$ & Low-dose $(n=10)$ & High-dose $(n=8)$ & P-value \\
\hline Age $(y)$ & $44.2 \pm 8.5$ & $52.1 \pm 7.4$ & $55.3 \pm 5.6$ & $0.020^{*}$ \\
\hline Age (y, median) & 40.5 & 54 & 56 & \\
\hline Female & $3(30)$ & $8(80)$ & $4(50)$ & 0.086 \\
\hline IBS subtype & & & & 0.245 \\
\hline Diarrhea & $6(60)$ & $9(90)$ & $5(62.5)$ & \\
\hline Constipation & $2(20)$ & $1(10)$ & $3(37.5)$ & \\
\hline Mixed & $2(20)$ & 0 & 0 & \\
\hline IBS duration (y) & $11.0 \pm 11.4$ & $10.8 \pm 7.2$ & $11.9 \pm 10.7$ & 0.966 \\
\hline Abdominal discomfort score & $5.8 \pm 1.9$ & $5.6 \pm 1.6$ & $4.6 \pm 1.2$ & 0.286 \\
\hline Abdominal bloating score & $5.7 \pm 2.2$ & $6.2 \pm 2.5$ & $4.8 \pm 1.7$ & 0.255 \\
\hline Formed stool frequency (per 10 times) & $2.6 \pm 1.6$ & $3.9 \pm 2.7$ & $4.1 \pm 2.7$ & 0.384 \\
\hline Epigastric soreness score & $3.3 \pm 2.5$ & $4.2 \pm 2.9$ & $3.0 \pm 3.5$ & 0.506 \\
\hline Nausea score & $1.0 \pm 1.9$ & $1.6 \pm 2.0$ & $1.4 \pm 1.4$ & 0.399 \\
\hline Fatigue Severity Scale & $44.4 \pm 7.0$ & $45.8 \pm 10.4$ & $47.4 \pm 11.4$ & 0.609 \\
\hline Fatigue Visual Analog Scale & $4.9 \pm 1.8$ & $4.8 \pm 1.9$ & $3.6 \pm 2.1$ & 0.256 \\
\hline Multidimensional Fatigue Inventory & $86.4 \pm 12.2$ & $90.0 \pm 12.6$ & $92.1 \pm 15.5$ & 0.438 \\
\hline White blood cell ( $\left.\times 10^{3} / \mathrm{uL}\right)$ & $6.8 \pm 0.8$ & $5.6 \pm 1.1$ & $5.8 \pm 1.0$ & 0.231 \\
\hline Hemoglobin $(\mathrm{g} / \mathrm{dL})$ & $14.7 \pm 1.5$ & $14.3 \pm 0.8$ & $14.0 \pm 1.4$ & 0.452 \\
\hline Hematocrit (\%) & $43.3 \pm 4.4$ & $43.9 \pm 3.3$ & $43.0 \pm 4.9$ & 0.960 \\
\hline Platelet $\left(\times 10^{3} / \mathrm{uL}\right)$ & $270.5 \pm 58.5$ & $240.0 \pm 43.6$ & $234.0 \pm 17.7$ & 0.612 \\
\hline Aspartate aminotransferase (U/L) & $26.8 \pm 5.1$ & $25.4 \pm 4.3$ & $27.5 \pm 7.7$ & 0.893 \\
\hline Alanine aminotransferase (U/L) & $21.9 \pm 7.8$ & $17.7 \pm 3.8$ & $15.9 \pm 4.9$ & 0.115 \\
\hline$\gamma$-Glutamyltranspeptidase (U/L) & $26.4 \pm 15.2$ & $19.4 \pm 7.1$ & $18.1 \pm 5.7$ & 0.569 \\
\hline Blood urea nitrogen (mg/dL) & $10.5 \pm 2.9$ & $13.3 \pm 4.1$ & $14.0 \pm 6.9$ & 0.492 \\
\hline Creatinine $(\mathrm{mg} / \mathrm{dL})$ & $0.9 \pm 0.2$ & $0.9 \pm 0.2$ & $0.9 \pm 0.1$ & 0.500 \\
\hline
\end{tabular}

Values are presented as mean \pm standard deviation or number (\%), unless otherwise stated. P-values from the Kruskal-Wallis test. IBS, irritable bowel syndrome.

${ }^{*} \mathrm{P}<0.05$. 
Table 2. Clinical variables of the study subjects at the last visit, 8 weeks after baseline $(n=28)$

\begin{tabular}{|c|c|c|c|c|}
\hline Variable & Placebo $(n=10)$ & Low-dose $(n=10)$ & High-dose $(n=8)$ & P-value \\
\hline Abdominal discomfort score & $5.4 \pm 2.3$ & $3.3 \pm 0.9$ & $2.0 \pm 1.1$ & $0.002^{*}$ \\
\hline Abdominal bloating score & $5.4 \pm 2.0$ & $4.3 \pm 1.8$ & $2.1 \pm 1.6$ & $0.006^{*}$ \\
\hline Formed stool frequency (per 10 times) & $3.4 \pm 1.6$ & $5.9 \pm 2.7$ & $7.4 \pm 2.3$ & $0.007^{\star}$ \\
\hline Epigastric soreness score & $2.9 \pm 2.1$ & $2.9 \pm 1.9$ & $1.8 \pm 2.2$ & 0.379 \\
\hline Nausea score & $1.3 \pm 1.5$ & $0.9 \pm 1.4$ & $0.4 \pm 0.5$ & 0.476 \\
\hline Fatigue Severity Scale & $42.9 \pm 8.3$ & $34.8 \pm 10.3$ & $34.0 \pm 9.6$ & 0.115 \\
\hline Fatigue Visual Analog Scale & $4.9 \pm 1.6$ & $3.5 \pm 2.2$ & $2.4 \pm 1.6$ & $0.028 *$ \\
\hline Multidimensional Fatigue Inventory & $86.0 \pm 12.7$ & $74.3 \pm 15.2$ & $73.4 \pm 13.6$ & $0.041^{*}$ \\
\hline White blood cell ( $\left.\times 10^{3} / \mathrm{uL}\right)$ & $8.0 \pm 1.9$ & $6.4 \pm 1.0$ & $6.5 \pm 1.0$ & 0.116 \\
\hline Hemoglobin $(g / d L)$ & $14.6 \pm 1.5$ & $14.2 \pm 0.7$ & $13.8 \pm 1.2$ & 0.382 \\
\hline Hematocrit (\%) & $44.5 \pm 4.2$ & $43.3 \pm 2.3$ & $41.5 \pm 3.2$ & 0.299 \\
\hline Platelet $\left(\times 10^{3} / \mathrm{uL}\right)$ & $232.8 \pm 42.2$ & $257.8 \pm 40.0$ & $220.0 \pm 32.4$ & 0.144 \\
\hline Aspartate aminotransferase (U/L) & $30.8 \pm 9.4$ & $29.5 \pm 11.1$ & $28.1 \pm 8.3$ & 0.720 \\
\hline Alanine aminotransferase (U/L) & $29.0 \pm 19.7$ & $23.3 \pm 14.7$ & $18.1 \pm 6.7$ & 0.359 \\
\hline$\gamma$-Glutamyltranspeptidase (U/L) & $28.4 \pm 18.0$ & $20.1 \pm 8.5$ & $18.4 \pm 6.1$ & 0.701 \\
\hline Blood urea nitrogen $(\mathrm{mg} / \mathrm{dL})$ & $12.0 \pm 2.0$ & $13.4 \pm 2.4$ & $15.8 \pm 5.2$ & 0.186 \\
\hline Creatinine $(\mathrm{mg} / \mathrm{dL})$ & $0.9 \pm 0.2$ & $0.8 \pm 0.1$ & $0.9 \pm 0.1$ & 0.181 \\
\hline
\end{tabular}

Values are presented as mean \pm standard deviation. P-values from the Kruskal-Wallis test.

${ }^{*} \mathrm{P}<0.05$.

Table 3. Comparison of clinical variables between study groups at 8 weeks $(n=28)$

\begin{tabular}{lccc}
\hline \multicolumn{1}{c}{ Variable } & Placebo vs. LD & Placebo vs. HD & LD vs. HD \\
\hline Abdominal discomfort & 0.058 & $0.002^{*}$ & 0.021 \\
Abdominal bloating & 0.187 & $0.003^{*}$ & 0.024 \\
Frequency of formed stool & 0.039 & $0.002^{*}$ & 0.244 \\
Fatigue Visual Analog Scale & 0.096 & $0.013^{*}$ & 0.197 \\
Multidimensional Fatigue Inventory & 0.028 & 0.037 & 0.721
\end{tabular}

Values are presented as the P-values from the Mann-Whitney test with Bonferroni correction.

LD, low-dose; HD, high-dose.

${ }^{*}$ P-values $<0.017$ were considered to indicate statistical significance by Bonferroni correction.

analysis. Frequency of formed stool showed a significant difference in the placebo, low-dose, and high-dose groups after intervention ( $\mathrm{P}=0.005,0.007$, and 0.010 , respectively) by $\mathrm{PP}$ analysis. In the ITT with LOCF analysis (the high-dose group only), none of these variables showed any significant difference, including abdominal discomfort $(\mathrm{P}=0.059)$ and frequency of formed stool $(\mathrm{P}=0.066)$. In the KruskalWallis test, abdominal discomfort, abdominal bloating, frequency of formed stool, fatigue VAS, and MFI showed statistical significance $(\mathrm{P}=0.002,0.006,0.007,0.028$, and 0.041 , respectively) in all three groups at 8 weeks (Table 2). However, after the Mann-Whitney test with Bonferroni correction, abdominal discomfort, abdominal bloating, frequency of formed stool, and fatigue VAS showed significant improvement in the high-dose group only $(\mathrm{P}=0.002,0.003,0.002$, and 0.013 , respectively) compared to the placebo group (Table 3 ). A statistically significant difference in FSS was not found between the groups at 8 weeks $(\mathrm{P}=0.115)$. In the ITT with LOCF analysis, none of the differences were statistically significant. The differences in five variables, abdominal discomfort, bloating, frequency of formed stool, fatigue VAS, and MFI, from baseline at 8 weeks also showed no statistical significance among the groups by $\mathrm{PP}$ analysis $(\mathrm{P}=0.497,0.253,0.125,0.194$,
Table 4. Spearman's correlation analysis between study groups at 8 weeks $(n=28)$

\begin{tabular}{lccccc}
\hline & $\begin{array}{c}\text { Abdominal } \\
\text { discomfort }\end{array}$ & $\begin{array}{c}\text { Abdominal } \\
\text { bloating }\end{array}$ & $\begin{array}{c}\text { Frequency of } \\
\text { formed } \\
\text { stool }\end{array}$ & $\begin{array}{c}\text { Fatigue } \\
\text { Visual } \\
\text { Analog Scale }\end{array}$ & $\begin{array}{c}\text { Multidimensional } \\
\text { Fatigue } \\
\text { Inventory }\end{array}$ \\
\hline$r$ & -0.668 & -0.604 & 0.605 & -0.513 & -0.408 \\
P-value & $<0.001^{*}$ & $0.001^{*}$ & $0.001^{*}$ & $0.005^{*}$ & $0.031^{*}$ \\
\hline
\end{tabular}

${ }^{*} \mathrm{P}<0.05$.

and 0.967 , respectively). Furthermore, there were no significant differences between the groups at 8 weeks with respect to white blood cell count and levels of hemoglobin, platelet count, AST, ALT, GGT, BUN, and creatinine $(\mathrm{P}=0.116,0.382,0.144,0.720,0.359,0.701,0.186$, and 0.181 by $\mathrm{PP}$ analysis, respectively).

\section{Correlations between Study Groups and Variables after Intervention}

Correlations between study groups and variables after intervention were determined by Spearman's rank correlation test (Table 4). Abdominal discomfort, abdominal bloating, formed stool frequency, fatigue VAS, and MFI showed significant correlations with the 3 study groups by $\mathrm{PP}$ analysis $(\mathrm{r}=-0.668, \mathrm{P}<0.001 ; \mathrm{r}=-0.604, \mathrm{P}=0.001 ; \mathrm{r}=0.605$, $\mathrm{P}=0.001 ; \mathrm{r}=-0.513, \mathrm{P}=0.005$; and $\mathrm{r}=-0.408, \mathrm{P}=0.031$, respectively). In the ITT with LOCF analysis, there were no significant correlations.

\section{Safety Assessment}

In this clinical trial, there were no reports of any significant adverse events during the study or any subjects experiencing either serious adverse events or withdrawal due to adverse events.

\section{DISCUSSION}

The present randomized, double-blind, and placebo-controlled study 
showed no significant dose-dependent clinical effects of synbiotics in IBS. However, significant positive effects of high-dose synbiotics on GI symptoms and fatigue were observed in IBS patients. In addition, the mean changes in abdominal discomfort, abdominal bloating, frequency of formed stool, fatigue VAS, and MFI scores showed a trend toward a dose-response relationship.

Recent studies have revealed many beneficial effects of probiotics or synbiotics on human health, especially the positive effects of probiotics on bowel symptoms in IBS patients.-10,14,15) In addition, emerging data have demonstrated the relationship between gut microorganisms and the nervous system, known as the gut-brain axis. ${ }^{16)} \mathrm{A}$ recent study elucidated that alteration of the intestinal microbiota, mucosal barrier disruption, and intestinal immune cell dysfunction collectively contribute to the pathogenesis of chronic fatigue syndrome. ${ }^{17)}$ Thus, administration of synbiotics can improve symptoms of fatigue as well as GI symptoms in IBS patients. Our study demonstrated that high-dose synbiotics were superior to placebo in improving the symptoms of IBS and fatigue.

To date, the exact pathogenesis of IBS remains unknown, but several mechanisms have been suggested to explain the efficacy of synbiotics in IBS, such as the influence of the intestinal luminal environment, the maintenance of epithelial and mucosal barrier function, and the modulation of the mucosal or systemic immune system including both innate and adaptive immune systems. ${ }^{10,18)}$ First, the restoration of a normal gut microbial environment may improve GI symptoms. Decreased normal gut flora and increased pathogenic microbiota have been found in IBS patients. ${ }^{19)}$ Such microbial imbalance erodes gut defense mechanisms and increases bacterial translocation, leading to diarrhea, bloating, and other GI symptoms. ${ }^{20)}$ Since synbiotics are effective in the restoration of normal gut flora and the defense against pathogenic organisms, it is possible that synbiotics will exert positive effects on symptoms via the recovery of the normal bowel environment in IBS. Second, the anti-inflammatory capability of synbiotics should be considered. A previous in vitro study has elucidated that synbiotics decrease inflammation in IBS. ${ }^{21)}$ Moreover, chronic inflammation is considered an important contributing factor to bowel symptoms. For example, inflammatory cytokines are increased in IBS patients and may be associated with GI symptoms. ${ }^{22)}$ In ulcerative colitis, increased inflammation reflects disease activity and correlates with severity of symptoms. ${ }^{23)}$ Inflammatory cytokines inhibit normal bowel functions, including water re-absorption by colonic villi, and bowel dysfunction may trigger bowel symptoms. ${ }^{24)}$ Therefore, the anti-inflammatory effects of synbiotics may result in improved GI symptoms in IBS patients.

The present study showed significant improvement in fatigue VAS after an 8-week treatment period with high-dose synbiotics. Fatigue may be considered in relation to gut microorganisms because gut microbial disruption induces biochemical and genetic changes in the brain associated with psychiatric conditions including anxiety and depression. ${ }^{25)}$ Indeed, administration of probiotics significantly reduces the recovery time from fatigue after forced swimming in rats. ${ }^{17)}$ However, until now, there exist only few data on the effect of synbiotics on fatigue in human studies; therefore, the precise role of synbiotics in
IBS-related mental problems should be investigated in the future.

In our data, only high-dose synbiotics were associated with significant improvement in IBS symptoms and fatigue compared with placebo. Previous studies on the optimal dose of synbiotics in IBS consist of four RCTs. ${ }^{14,15,26,27)}$ Of these, three studies indicated positive results and one showed a negative result. This discrepancy may be due to variations in inclusion criteria for the subjects, sample size, study design, and synbiotic dosage. ${ }^{28)}$ Of these heterogeneous factors, our study suggests that dosage plays an important role in the therapeutic effects of IBS. This finding may also be supported by our data. Although no significant dose-dependent clinical effects of synbiotics were observed, a trend toward a dose-response relationship was observed.

Our study has some limitations. First, since the sample size of the current study was small, we used nonparametric analysis. In addition, we were unable to perform subgroup analysis by IBS subtypes because of the small sample size. A large-scale prospective study should be performed to investigate the efficacy of synbiotics on bowel symptoms and fatigue in IBS subtypes. Second, the direct measurement of fecal microbial levels for the restoration of normal flora after synbiotic supplementation has not been performed. However, in previous studies, it has been shown that probiotics and prebiotics were able to modify the gut flora in healthy volunteers. ${ }^{29,30)}$ Finally, an 8-week study period may be too short to confirm the long-term benefits and side effects of synbiotics.

Otherwise, our study is another well-designed RCT examining the efficacy and safety of synbiotics in IBS that used fatigue scales to assess the degree of fatigue symptoms in IBS. Moreover, our study has the strength of being the first to evaluate the dose-response relationship between synbiotics and gut symptoms and fatigue in IBS.

In summary, this study indicates that high-dose synbiotics containing Lactobacillus, Bifidobacterium, and fructooligosaccharides are effective and safe for the treatment of abdominal discomfort, abdominal bloating, formed stool frequency, and fatigue symptoms in IBS. In addition, although were no significant dose-dependent clinical effects of synbiotics were noted, a trend toward a dose-response relationship was demonstrated. In the future, further large-scale studies to determine the association between optimal dosage of synbiotics and IBS are warranted.

\section{CONFLICT OF INTEREST}

No potential conflict of interest relevant to this article was reported. Since the current research was an investigator-initiated trial, the study design, randomization, and data collection and analysis were performed by the investigators. The study was supported by a research grant and supply of the placebo and the study drug from P\&P Holdings, LTD (Seoul, Korea).

\section{ACKNOWLEDGMENTS}

This work was supported by a research grant from P\&P Holdings, LTD 
(Seoul, Korea).

\section{REFERENCES}

1. Longstreth GF, Thompson WG, Chey WD, Houghton LA, Mearin F, Spiller RC. Functional bowel disorders. Gastroenterology 2006;130: 1480-91.

2. Han SH, Lee OY, Bae SC, Lee SH, Chang YK, Yang SY, et al. Prevalence of irritable bowel syndrome in Korea: population-based survey using the Rome II criteria. J Gastroenterol Hepatol 2006;21:1687-92.

3. Spiegel BM. Burden of illness in irritable bowel syndrome: looking beyond the patient. Clin Gastroenterol Hepatol 2013;11:156-7.

4. Parkes GC, Brostoff J, Whelan K, Sanderson JD. Gastrointestinal microbiota in irritable bowel syndrome: their role in its pathogenesis and treatment. Am J Gastroenterol 2008;103:1557-67.

5. Ghoshal UC, Shukla R, Ghoshal U. Small intestinal bacterial overgrowth and irritable bowel syndrome: a bridge between functional organic dichotomy. Gut Liver 2017;11:196-208.

6. Brenner DM, Moeller MJ, Chey WD, Schoenfeld PS. The utility of probiotics in the treatment of irritable bowel syndrome: a systematic review. Am J Gastroenterol 2009;104:1033-49.

7. McFarland LV, Dublin S. Meta-analysis of probiotics for the treatment of irritable bowel syndrome. World J Gastroenterol 2008;14:2650-61.

8. Kim HJ, Camilleri M, McKinzie S, Lempke MB, Burton DD, Thomforde GM, et al. A randomized controlled trial of a probiotic, VSL\#3, on gut transit and symptoms in diarrhoea-predominant irritable bowel syndrome. Aliment Pharmacol Ther 2003;17:895-904.

9. Zeng J, Li YQ, Zuo XL, Zhen YB, Yang J, Liu CH. Clinical trial: effect of active lactic acid bacteria on mucosal barrier function in patients with diarrhoea-predominant irritable bowel syndrome. Aliment Pharmacol Ther 2008;28:994-1002.

10. Michail S, Kenche H. Gut microbiota is not modified by randomized, double-blind, placebo-controlled trial of VSL\#3 in diarrhea-predominant irritable bowel syndrome. Probiotics Antimicrob Proteins 2011;3: 1-7.

11. Riegler G, Esposito I. Bristol scale stool form: a still valid help in medical practice and clinical research. Tech Coloproctol 2001;5:163-4.

12. Krupp LB, LaRocca NG, Muir-Nash J, Steinberg AD. The fatigue severity scale: application to patients with multiple sclerosis and systemic lupus erythematosus. Arch Neurol 1989;46:1121-3.

13. Smets EM, Garssen B, Bonke B, De Haes JC. The Multidimensional Fatigue Inventory (MFI) psychometric qualities of an instrument to assess fatigue. J Psychosom Res 1995;39:315-25.

14. Tsuchiya J, Barreto R, Okura R, Kawakita S, Fesce E, Marotta F. Singleblind follow-up study on the effectiveness of a symbiotic preparation in irritable bowel syndrome. Chin J Dig Dis 2004;5:169-74.

15. Min YW, Park SU, Jang YS, Kim YH, Rhee PL, Ko SH, et al. Effect of composite yogurt enriched with acacia fiber and Bifidobacterium lactis. World J Gastroenterol 2012;18:4563-9.

16. Mayer EA. Gut feelings: the emerging biology of gut-brain communication. Nat Rev Neurosci 2011;12:453-66.

17. Singh PK, Chopra K, Kuhad A, Kaur IP. Role of Lactobacillus acidophilus loaded floating beads in chronic fatigue syndrome: behavioral and biochemical evidences. Neurogastroenterol Motil 2012;24:366-e170.

18. Forsythe P, Bienenstock J. Immunomodulation by commensal and probiotic bacteria. Immunol Invest 2010;39:429-48.

19. Zhuang X, Xiong L, Li L, Li M, Chen M. Alterations of gut microbiota in patients with irritable bowel syndrome: a systematic review and meta-analysis. J Gastroenterol Hepatol 2017;32:28-38.

20. Guarner F, Malagelada JR. Gut flora in health and disease. Lancet 2003;361:512-9.

21. Grimoud J, Durand H, de Souza S, Monsan P, Ouarne F, Theodorou V, et al. In vitro screening of probiotics and synbiotics according to antiinflammatory and anti-proliferative effects. Int J Food Microbiol 2010; 144:42-50.

22. De Giorgio R, Barbara G. Is irritable bowel syndrome an inflammatory disorder? Curr Gastroenterol Rep 2008;10:385-90.

23. Muzes G, Molnar B, Tulassay Z, Sipos F. Changes of the cytokine profile in inflammatory bowel diseases. World J Gastroenterol 2012;18: 5848-61.

24. Barbara G, Cremon C, Carini G, Bellacosa L, Zecchi L, De Giorgio R, et al. The immune system in irritable bowel syndrome. J Neurogastroenterol Motil 2011;17:349-59.

25. Benton D, Williams C, Brown A. Impact of consuming a milk drink containing a probiotic on mood and cognition. Eur J Clin Nutr 2007; 61:355-61.

26. Rogha M, Esfahani MZ, Zargarzadeh AH. The efficacy of a synbiotic containing Bacillus Coagulans in treatment of irritable bowel syndrome: a randomized placebo-controlled trial. Gastroenterol Hepatol Bed Bench 2014;7:156-63.

27. Abbas Z, Yakoob J, Jafri W, Ahmad Z, Azam Z, Usman MW, et al. Cytokine and clinical response to Saccharomyces boulardii therapy in diarrhea-dominant irritable bowel syndrome: a randomized trial. Eur J Gastroenterol Hepatol 2014;26:630-9.

28. Ford AC, Quigley EM, Lacy BE, Lembo AJ, Saito YA, Schiller LR, et al. Efficacy of prebiotics, probiotics, and synbiotics in irritable bowel syndrome and chronic idiopathic constipation: systematic review and meta-analysis. Am J Gastroenterol 2014;109:1547-61.

29. Elli M, Callegari ML, Ferrari S, Bessi E, Cattivelli D, Soldi S, et al. Survival of yogurt bacteria in the human gut. Appl Environ Microbiol 2006;72:5113-7.

30. Elli M, Cattivelli D, Soldi S, Bonatti M, Morelli L. Evaluation of prebiotic potential of refined psyllium (Plantago ovata) fiber in healthy women. J Clin Gastroenterol 2008;42 Suppl 3 Pt 2:S174-6. 